\title{
Evaluation of the QIAstat-Dx Respiratory SARS-CoV-2 Panel for early diagnosis of COVID-19
}

\section{Dear Editor,}

An effective response to the SARS-CoV-2 that has caused the coronavirus disease 2019 (COVID-19) pandemic $^{1}$ requires rapid and accurate diagnostic testing. We evaluate the QIAstat-Dx ${ }^{\circledR}$ Respiratory SARS-CoV-2 Panel-a multiplex real-time polymerase chain reaction (RT-PCR) assay-against an E-gene RT-PCR assay ${ }^{2}$ that successfully identified cases at the start of the COVID-19 pandemic in Singapore. ${ }^{3}$ The QIAstat-Dx is a cartridge-based assay that integrates sample ribonucleic acid (RNA) extraction, reverse transcription, and a multiplex RT-PCR assay against 22 different respiratory pathogens (including SARS-CoV-2) in 1 self-contained system. ${ }^{4-7}$ For the SARS-CoV-2 analyte, the assay targets both the E-gene and open reading frame 1a (ORF1a) regions of the viral genome. Prior work comparing different sets of primers and probes found that this E-gene primer combination was one of the most sensitive. ${ }^{8}$

This study received approval for the use of residual diagnostic samples, as well as a waiver of requirements for patient informed consent, from the Singapore General Hospital Institutional Review Board (\#2020/2138).

We first compared the analytical limits of detection (LoD) of the QIAstat-Dx against the E-gene RT-PCR assay. A quantitated pool of SARS-CoV-2 positive oropharyngeal swab samples was serially diluted and run on both platforms. Probit regression analysis was used to calculate the theoretical limits of detection for each assay. The E-gene RT-PCR LoD was 223 E-gene copies/reaction and the QIAstat-Dx LoD was 193 E-gene copies/reaction, with no statistically significant difference at the $95 \%$ confidence level.

Next, we tested the analytical sensitivity of the QIAstat-Dx by assessing its ability to detect SARSCoV-2 infection on archived oropharyngeal swab samples. The first positive samples of 40 confirmed COVID-19 patients detected on our E-gene RT-PCR assay were run on the QIAstat-Dx. These samples best represent swabs obtained when acutely infected patients present for medical care. The QIAstat-Dx reported 38 out of the 40 samples as positive, giving a diagnostic sensitivity of at least 95\%. The 2 missed samples had high cycle threshold (Ct) values of 31.7 and 36.3, respectively, and the viral RNA may have inadvertently degraded with the freeze-thaw cycle inherent in using archived samples. Among the 40 samples, the $\mathrm{Ct}$ values ranged 13.4-30.0.

Lastly, we assessed the QIAstat-Dx for diagnostic specificity and cross-reactivity against other common respiratory tract pathogens. Twenty samples negative for SARS-CoV-2 were run on the QIAstat-Dx platform (Table 1). The QIAstat-Dx did not cross-react with the common respiratory tract pathogens tested, and did not react with known negative samples, giving a diagnostic specificity of $100 \%$.

Our study was limited by the global shortage of testing reagents and the need to prioritise clinical testing. Within these limitations, the QIAstat-Dx demonstrated good overall performance for the SARSCoV-2 analyte, with a diagnostic sensitivity of $95 \%$ (38/40 positive samples) and diagnostic specificity of $100 \%$ (20/20 negative samples). There was no crossreactivity with the other common respiratory pathogens tested. In vitro studies by the manufacturer claim no cross-reactivity with SARS-CoV, available in the QIAstat-Dx Respiratory SARS-CoV-2 Panel Instructions for Use (Handbook, March 2020). The analytical limit of detection was similar to our existing E-gene RT-PCR assay. Our findings are consistent with a separate QIAstat-Dx study using prospectively collected nasopharyngeal swabs. ${ }^{4}$

The self-contained cartridge format of the QIAstat-Dx offers several advantages over conventional RT-PCR assays. It is easy to use, with no separate viral RNA extraction, reverse transcription and RT-PCR steps. This requires less training compared with conventional RT-PCR assays. Furthermore, having fewer handling steps reduces the risk of sample contamination. The run time of 70 minutes is similar to other rapid PCR assays, compared with an average of 3 hours for conventional RT-PCR assays. Also, the ability to test for other common respiratory pathogens that present similarly to SARS-CoV-2 allows the clinician to quickly identify the causative agent.

The major drawback of the QIAstat-Dx platform is the assay throughput. A 1-module instrument is only able to run 1 sample at a time; additional modules are required to run more samples simultaneously.

Overall, the QIAstat-Dx Respiratory SARS-CoV-2 Panel seems best suited to small numbers of urgent 
Table 1. QIAstat-Dx Respiratory SARS-CoV-2 Panel does not cross-react with other common respiratory pathogens tested

\begin{tabular}{lcc}
\hline Sample no. & Known result & QIAstat-Dx result \\
\hline A & Influenza A & Influenza A (H3) \\
\hline B & Human Metapneumovirus & Human Metapneumovirus A/B \\
\hline C & Coronavirus 229E Influenza A (low positive) & Coronavirus 229E \\
\hline D & Coronavirus OC43/HKU1 & Coronavirus HKU1 \\
\hline E & Coronavirus NL63 & Coronavirus NL63 \\
\hline F & Mycoplasma pneumoniae & Mycoplasma pneumoniae \\
\hline G & Influenza B & Influenza B \\
\hline H & Rhinovirus & Rhinovirus/Enterovirus \\
\hline I & Adenovirus & Adenovirus \\
\hline J & Parainfluenza Virus 1 & Parainfluenza Virus 1 \\
\hline K to T & No pathogens detected & No pathogens detected \\
\hline
\end{tabular}

samples, when concurrent detection of other respiratory pathogens is desired. Rapidly identifying respiratory pathogens allows for COVID-19 patients to be isolated quickly, while also offering early diagnosis for other patients with respiratory infections.

\section{Acknowledgements}

The authors thank QIAGEN GmbH, Dr Gavian Lua, Eneko Goya and Dr Josep Pareja for their technical assistance and supply of cartridges. Funding was partially covered by the Singapore General Hospital Research Grant SRG-NIG-04-2019.

\section{REFERENCES}

1. Zhu N, Zhang D, Wang W, et al. A Novel Coronavirus from Patients with Pneumonia in China, 2019. N Engl J Med 2020;382:727-33.

2. Corman VM, Landt O, Kaiser M, et al. Detection of 2019 novel coronavirus (2019-nCoV) by real-time RT-PCR. Euro Surveill 2020;25:2000045

3. Young BE, Ong SWX, Kalimuddin S, et al. Epidemiologic Features and Clinical Course of Patients Infected With SARS-CoV-2 in Singapore. JAMA 2020;323:1488-94.

4. Visseaux B, Le Hingrat Q, Collin G, et al. Evaluation of the QIAstat-Dx Respiratory SARS-CoV-2 Panel, the first rapid multiplex PCR commercial assay for SARS-CoV-2 detection. J Clin Microbiol 2020;58:e0630-20.
5. Boers SA, Melchers WJG, Peters CJA, et al. Multicenter evaluation of the QIAstat-Dx Respiratory Panel V2 for the detection of viral and bacterial respiratory pathogens. J Clin Microbiol 2020;58:e01793-19.

6. Parčina M, Schneider UV, Visseaux B, et al. Multicenter evaluation of the QIAstat Respiratory Panel-A new rapid highly multiplexed PCR based assay for diagnosis of acute respiratory tract infections. PLoS One 2020;15:e230183.

7. Leber AL, Lisby JG, Hansen G, et al. Multicenter Evaluation of the QIAstat-Dx Respiratory Panel for the Detection of Viruses and Bacteria in Nasopharyngeal Swab Specimens. J Clin Microbiol 2020;58:e0155-20.

8. Nalla AK, Casto AM, Huang MW, et al. Comparative Performance of SARS-CoV-2 Detection Assays using Seven Different Primer/Probe Sets and One Assay Kit. J Clin Microbiol 2020;58:e0557-20.

Kenneth C $\underline{\text { Goh }},{ }^{* 1,2}$ PhD, Shireen YL Tan, ${ }^{{ }^{1}, 2}$ MB BChir PhD, Wan Loo Tan, ${ }^{1} M S c$, Kun Lee Lim, ${ }^{1} P h D$,

Karrie KK 으, ${ }^{1,2}{ }_{F R C P A}$, Lynette Lin-Ean Oon, ${ }^{1}$ FRCPA, Kian Sing Chan, ${ }^{1}$ FRCPA

'Department of Molecular Pathology, Singapore General Hospital, Singapore ${ }^{2}$ Department of Microbiology, Singapore General Hospital, Singapore

Correspondence: Dr Kenneth C Goh, Department of Microbiology, Singapore General Hospital, 20 College Road, Level 7, Singapore 169856. Email: kenneth.goh@mohh.com.sg

* Joint first authors 